\title{
The effects of severe aortic stenosis and high pulmonary artery pressure on aortic valve replacement ${ }^{*}$
}

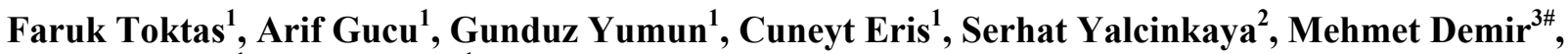 \\ Tuğrul Goncu ${ }^{1}$, Senol Yavuz ${ }^{1}$
}

${ }^{1}$ Cardiovascular Surgery Department, Bursa Yuksek Ihtisas Training and Research Hospital, Bursa, Turkey

${ }^{2}$ Thoracic Surgery Department, Bursa Yuksek Ihtisas Training and Research Hospital, Bursa, Turkey

${ }^{3}$ Cardiology Department, Bursa Yuksek Ihtisas Training and Research Hospital, Bursa, Turkey

Email: "drmehmetd@gmail.com

Received 2 July 2013; revised 12 August 2013; accepted 1 September 2013

Copyright (C) 2013 Faruk Toktas et al. This is an open access article distributed under the Creative Commons Attribution License, which permits unrestricted use, distribution, and reproduction in any medium, provided the original work is properly cited.

\begin{abstract}
Background: Pulmonary hypertension development in pure severe aortic stenosis is a situation that affects mortality and morbidity. Material and Methods: Data from files of 31 patients with systolic pulmonary artery pressure over $50 \mathrm{~mm} \mathrm{Hg}$ and with pure severe aortic stenosis, and underwent aortic valve replacement in our clinic were examined retrospectively. Results: Preoperative effort capacities of the patients were evaluated as follows according to NYHA; 4 patients class 1-2, 16 patients class 3 , and 11 patients class 4. Twenty-five metal, and 6 biologic aortic valves were used. Postoperative hospital mortality was recorded as $12 \%$ with 4 patients. Patients were reevaluated on the postoperative 2nd and 12th months. Pulmonary arterial pressure of the patients was measured using echocardiography. Preoperative average pulmonary artery systolic pressure was measured as $55 \pm 3 \mathbf{~ m m H g}$. The average pressure was measured as $41 \pm 3 \mathrm{mmHg}$ on the $2 \mathrm{nd}$, and as $37.8 \pm 4 \mathrm{mmHg}$ on the 12th month. The effort capacity evaluation in the postoperative 2 nd month was as follows: 11 patients class 1-2, 12 patients class 3 , and 4 patients class 4 . The effort capacity evaluation conducted in the 12th month was: 14 patients were class $1-2,10$ patients were class 3 , and 2 patients were class 4 . During the follow-up 1 year, survival rate of the patients was determined as $83.8 \%$ average, 5 year survival rate was determined as $61.5 \%$. Conclusion: We believe that AVR may be performed in severe aortic stenosis cases with high pulmonary pressure with acceptable mortality, leading to a better quality and longer life.
\end{abstract}

\footnotetext{
"Conflict of interest: none declared.

${ }^{\#}$ Corresponding author.
}

Keywords: Aortic Valve Stenosis; Pulmonary Hypertension; Heart Valve Prosthesis Implantation; Prognosis

\section{INTRODUCTION}

Cases with aortic valve area below $0.6 \mathrm{~cm}^{2} / \mathrm{m}^{2}$ are accepted as severe aortic stenosis (AS) [1,2]. In several publications, it is stated that the natural course of severe AS is significantly bad [3-5]. Presence of pulmonary hypertension $(\mathrm{PH})$ in severe aortic stenosis is a prognosis negative indicator [6]. The negative prognosis indicators limiting surgery include: narrow aortic valves, left ventricle function disorder, severe aortic stenosis with low aortic valve gradient, severe aortic stenosis with pulmonary hypertension development, and aortic stenosis with cardiac arrhythmia [6-8]. Among these risk factors, lesser and incomprehensive series were published in the literature in regards to implementation of aortic valve replacement (AVR) in pure severe aortic stenosis with pulmonary hypertension when compared to the other factors.

In our study, we aimed to assess the effect of pulmonary hypertension development in severe aortic stenosis on aortic valve surgery results.

\section{PATIENTS AND METHODS}

Data from the files of the cases underwent aortic valve replacement with systolic pulmonary pressure over 50 $\mathrm{mmHg}$ and with pure severe aortic stenosis, in our clinic between the years of 1996 and 2011 were examined retrospectively.

The inclusion criteria were:

1) Severe aortic stenosis and pulmonary artery systolic pressure over $50 \mathrm{mmHg}$ due to aortic stenosis. 
2) Tricuspid regurgitation flow rate higher than 3.8 $\mathrm{m} / \mathrm{sn}$.

3) No evidence of additional valve disease except for tricuspid insufficiency developing secondary to pulmonary hypertension. [While selecting, cases with mitral narrowness (valve area larger than $2.5 \mathrm{~cm}^{2}$ ), more than first degree mitral insufficiency) or, more than first degree aortic regurgitation were not included in the study group].

4) No accompanying congenital anomalies.

Patients with former valve surgery, and/or previous cardio-vascular surgery, were excluded. Those with accompanying coronary lesions were included in the study. During the same session proper grafts were placed in the required veins and by-pass was conducted. Patients with developed tricuspid insufficiency were included in the study. During the same session De Vega annuloplasty and proper tricuspid annuloplasty were applied to necessary patients.

\section{OPERATIVE TECHNIQUE}

General anesthesia was administered to the patients through standard balance anesthesia technique. Propofol, and dormicum were preferred for the induction of the patients. Norcuron and/or pancuronium were used as muscular relaxant. Inhalation anesthetics were used when required and isofloran, and/or sevorein were preferred. Fentanyl citrate was used as narcotic analgesic. Upon anesthesia induction right cardiac catheterization was implemented in all patients with swan-ganz catheter (model 131H-7F; Baxter Healthcare Corp., Edwards Div., Irvine, Calif.). Pulmonary artery pressure was monitored. Heparin was given at $3 \mathrm{mg} / \mathrm{kg}$ dose, and heparinization was performed as ACT (activated clotting time) over 480 seconds. During our operations (Pemco Inc., Cleveland, OH, USA) Roller pump, (membranous oxygenator) hollow-fiber membrane oxygenator (Edwards Vitale ${ }^{\mathrm{TM}}$, Edwards Lifesciences LLC, One Edwards Way, Irvine, CA, USA) were used. Medium level hypothermia was used in the patients (average $30^{\circ} \mathrm{C}-32^{\circ} \mathrm{C}$ ). In cardiac arrest (Plegisol, Abbott Laboratories, North Chicago, IL) crystalloid cardioplegia was used. For maintenance cardiac arrest was continued through cold blood cardioplegia with intermittent potassium and sodium bicarbonate addition in antegrade way with 20 minute intervals.

After standard cannulation process, cardiopulmonary bypass was initiated and aortic valve replacement was performed by single stitches by using suture technique with pledgets 2/0 braided polyester non absorbable stitch.

\subsection{Statistical Analysis}

Statistical evaluation was conducted by using SPSS 9.0 programs. Student's t test and $X^{2}$ tests were used in ana- lyzing variances. Kaplan-Meier analysis was used for survival expectation analysis. A p value $<0.05$ was accepted as significant.

\subsection{Findings}

There were 31 patients meeting the inclusion criteria. Twelve of these patients were female (38.7\%), and the remaining 19 were male (62.3\%). Mean ages of the patients was $53.0 \pm 2.3$ years (range 38 - 79). Demographic characteristics of the patients are given in Table $\mathbf{1}$.

Preoperative effort capacities of the patients were evaluated as follows according to NYHA: 4 patients class 1-2, 16 patients class 3, and 11 patients class 4 .

During operation, 25 metal aortic valves varying between 19 - 25 in size from various manufacturers (e.g. Carbomedix, St-Jude Medical, and Medtronic), and 6 biologic aortic valves were used. In 5 patients, Manouguian procedure for aortic root enlargement was performed due to narrow aortic root. In 12 patients with coronary artery disease, coronary artery bypass grafts were added to procedure during the same session. De Vega Tricuspid annuloplasty was performed using 2/0 prolene in 12 patients with tricuspid insufficiency at 3 - 4 level.

We did not have any perioperative mortality. In one patient with additional coronary bypass grafting, we needed intra-aortic balloon pump for pump retrieval.

Average intubation period was $4.5 \pm 1$ hours. In two cases we had a prolonged intubation period exceeding 48 hours.

Table 1. Demographic characteristics of the patients.

\begin{tabular}{|c|c|}
\hline Demographic characteristics of the patients & Values \\
\hline Age (average) & $38-79(53.0+2.3)$ \\
\hline Gender male/female & $12 / 19$ \\
\hline Hypertension & $60 \%$ \\
\hline Diabetes & $17 \%$ \\
\hline Coronary artery disease & $38 \%$ \\
\hline Chest pain (angina) & $55 \%$ \\
\hline Renal failure (creatine > 1.8) & $27 \%$ \\
\hline Congestive heart failure & $55 \%$ \\
\hline Chronic obstructive pulmonary disease (COAH) & $12 \%$ \\
\hline Aspirin use & $52 \%$ \\
\hline Beta blocker use & $22 \%$ \\
\hline Angiotensin converting enzyme inhibitor use & $38 \%$ \\
\hline Statin use & $27 \%$ \\
\hline Pulmonary artery systolic pressure (mmHg) & $55 \pm 3$ \\
\hline
\end{tabular}


Pulmonary arterial systolic pressure measurements of all patients were conducted by swan-ganz catheter placed upon general anesthesia administration. Measurement was repeated on the postoperative 24th hour. Pulmonary arterial systolic pressure measurements and changes are given in Table 2. Preoperative average pulmonary arterial systolic pressure measurements value was measured as $55 \pm 3 \mathrm{mmHg}$. There was a difference between the measurement at preoperative catheter laboratory and preoperative operation table. Surgery room measurements were taken under general anesthesia, following a 6-hour period of starving, therefore these measurements were lower. Thus, we used the values measured during angiography in the study. The postoperative 24 hours average pulmonary arterial systolic pressure measurements value of the patients was determined as $43 \pm 5$ mmHg. Variance was calculated as $25.38 \%$ (p < 0.001).

Hospital mortality was experienced in 4 patients $(12 \%)$. In two cases the cause of death were the prolonged intubation due to respiratory failure, and accompanying multiple organ failure. One case died during laparatomy secondary to mesenteric ischemia, and severe intestinal resection. One case died due to low cardiac flow.

During the 12-month follow-up, another case died due to extra cardiac reasons.

Patients were reevaluated in the postoperative 2nd and 12th months. Pulmonary arterial systolic pressure of the patients were measured using echocardiography (Vivid 7, GE Medical Systems, Vingmed, Horton, Norway). The average pulmonary artery systolic pressure were measured as $41 \pm 3 \mathrm{mmHg}$ on the 2nd month, and as $37.8 \pm 4$ $\mathrm{mmHg}$ on the 12th month, respectively.

The average follow-up was 5.2 patient years, and $88.4 \%$ of the patients were followed between 6 months and 13 years. During the follow up period, the 1-year survival rate was determined as $83.8 \%$, and 5 -year sur- vival rate was determined as $61.5 \%$.

Ejection fractions of the patients were measured on preoperative, early postoperative (within 2 weeks following surgery), postoperative 2nd month, and 12th month periods. While the preoperative ejection fraction average was $38 \% \pm 18 \%$, this value was obtained as $42 \%$ $\pm 23 \%$ during early postoperative period, as $43 \% \pm 20 \%$ at the 2 nd month, and as $46.6 \% \pm 3 \%$ at the 12 th month (Table 2).

Patients were evaluated according to NYHA criteria in terms of effort capacities during the same period. Results obtained in the effort capacity evaluation during postoperative 2nd month were as follows: 11 patients class 1-2, 12 patients class 3 , and 4 patients class 4 . During the effort capacity evaluation at the 12th month 14 patients were class $1-2,10$ patients were class 3 , and 2 patients were class 4 .

\section{DISCUSSION}

Cases below $0.6 \mathrm{~cm}^{2} / \mathrm{m}^{2}$ with valve area in aortic narrowness are accepted as severe aortic stenosis [1,2]. Various publications are available reporting on high mortality rates for severe aortic stenosis cases when valve replacement is not conducted [9-11]. Congestive heart failure emerges significantly in severe aortic stenosis cases within average two years [4]. In AS cases, left ventricle hypertrophy is the reason for severe cardiac arrhythmia and sudden death [12]. Low left ventricle ejection fraction in complex and frequent ventricle arrhythmias and increased peak systolic wall stress are common in Cardiac arrhythmias [13,14]. An important determinant of sudden death in aortic stenosis is the pulmonary hypertension developed parallel to left ventricle function disorder [15]. Positive effect of AVR on patient survival and ejection fraction in severe aortic stenosis are revealed in several studies [16,17]. Sig-

Table 2. Preoperative postoperative measurements and variances of the patients.

\begin{tabular}{|c|c|c|c|c|c|c|}
\hline Measured values & $\begin{array}{c}\text { Preoperative } \\
\text { average } \\
\text { values } n=31\end{array}$ & $\begin{array}{c}\text { Postoperative } \\
\text { early period } \\
\text { average value }\end{array}$ & $\begin{array}{l}\text { Postoperative } \\
\text { second month } \\
\text { average value } \\
\quad \mathrm{n}=27 \\
\end{array}$ & $\begin{array}{l}\text { Postoperative } \\
\text { twelfth month } \\
\text { average value } \\
\quad n=26 \\
\end{array}$ & $\begin{array}{c}\text { Preoperative } \\
\text { measurement-postoperative } \\
\text { 12th month measurement } \\
\text { variance rate }\end{array}$ & p value \\
\hline Pulmonary artery systolic pressure (mmHg) & $58.3 \pm 7$ & $43.5 \pm 5$ & $41.3 \pm 3$ & $37.8 \pm 4$ & $35.16 \%$ & $\mathrm{p}<0.001$ \\
\hline Injection fraction \% & $34 \pm 4.8$ & $42 \pm 2.3$ & $43 \pm 2.1$ & $46 \pm 3.4$ & $34.59 \%$ & $\mathrm{p}<0.001$ \\
\hline Aortic valve area $\left(\mathrm{cm}^{2}\right)$ & 0.68 & & & & & \\
\hline Aortic gradient (mmHg) & $68 \pm 16$ & & 22 & 21 & $69.19 \%$ & $\mathrm{p}<0.001$ \\
\hline Septum thickness $(\mathrm{cm})$ & $1.5 \pm 0.2 \mathrm{~cm}$ & & $1.46 \pm 0.3 \mathrm{~cm}$ & $1.38 \pm 0.2 \mathrm{~cm}$ & $8 \%$ & $\mathrm{p}<0.01$ \\
\hline Posterior wall thickness $(\mathrm{cm})$ & $1.2 \pm 0.1 \mathrm{~cm}$ & & $1.18 \pm 0.2 \mathrm{~cm}$ & $1.16 \pm 0.2 \mathrm{~cm}$ & $0.4 \%$ & $\mathrm{p}<0.001$ \\
\hline Left ventricle end-diastolic diameter $(\mathrm{cm})$ & $5.2 \pm 0.8$ & & & $4.7 \pm 0.8$ & $9.6 \%$ & $\mathrm{p}<0.001$ \\
\hline Tricuspid regurgitation flow rate & $3.97 \pm 0.5$ & & & $2.7 \pm 0.8$ & $29.97 \%$ & $\mathrm{p}<0.001$ \\
\hline
\end{tabular}


nificant recovery observed in our study results in terms of patient ejection fractions. While the preoperative ejection fraction average was $38 \% \pm 18 \%$, this value was obtained as $42 \% \pm 23 \%$ during early postoperative period, as $43 \% \pm 20 \%$ at the 2 nd month, and as $46.6 \% \pm$ $3 \%$ at the 12 th month.

Johnson et al. [18] published the first AVR experience in severe aortic stenosis cases with severe pulmonary hypertension in 1988. Later Tracy [19] and Snopek [20] published the series which were not comprehensive and with low mortality results. More extensive case series and randomized studies were conducted by Malouf et al. In his study, AVR was performed on 37 patients with severe aortic stenosis cases developed severe pulmonary hypertension were compared to AVR not-implemented on 10 patients. During the study, operative mortality was reported as 6 (16\%), and late mortality was reported as $9 \%$; total 32\% mortalities were reported. Eight of the patients treated by conservative treatment died (80\%). In our study mortality was $12 \%$ and is in accordance with the mortality rate of severe aortic stenosis with left ventricle function disorder reported in the literature $[8,17,21]$.

In the comprehensive study conducted by Ramdas et al. [22] AVR with conducted group and AVR without conducted group of patients with average age 75 , average pulmonary artery pressure $69 \mathrm{mmHg}$ with severe aortic stenosis were compared. In AVR not conducted group, 1-month mortality was reported $30 \%$ and 1-year mortality was determined as $70 \%$. Renal failure, old age, heart failure, dementia, low ejection fraction were noted as the important causes of mortality. In the same study 1-month mortality rate of AVR conducted group of patients was $8 \%$, 5-year mortality rate was determined as $65 \%$. For AVR not conducted patients, 5-year survival rate was $20 \%$. These significantly successful results display the beneficial results of AVR. However, we believe that the case of Ramdas et al. [22] including the mitral valve attempts in addition to aortic stenosis is an important detail. Implemented mitral valve attempts might affect the reveal of the AVR effect on pulmonary hypertension regression.

During the follow-up of medium and severe aortic stenosis cases with no AVR implementation conducted by Chizner et al. [3], mortality speed was determined as follows: $26 \%$ for the 1 st year, $48 \%$ for the 2 nd year, and $57 \%$ for the 3rd year. In various studies, 5-year mortality rate differs between $18 \%$ - 50\% follow-ups of 453 patients with severe aortic stenosis with no AVR implementation conducted by Padmini [23] et al. The survival rates of 1 year, 5 years and 10 years were found respectively as $62 \%, 32 \%$, and $18 \%$. In our study, 1 -year survival rate was $83.8 \%$ and 5-year survival rate was calculated as $61.5 \%$.

Long follow-up period and long follow-up results of studies conducted by Ramdas et al. [22] and Malouf et al. [21] are close to our study, however, the average age is higher than our study group. The most important reason why patient average age in our study is younger than that of other studies is that we face cardiac valve diseases earlier than Western societies due to common rheumatic heart diseases in our population. This situation seems to be the reason why the average age of the patient population included in our study is much younger than the series in the literature. (While the etiology of the aortic valve disease in our patient group is mostly formed by rheumatic heart disease, senile degeneration is observed as the most etiologic factor in the series included in the literature.) Moreover, survival length of our patients can be explained by employing relatively younger patient population.

Today cardiac surgery increases in number and finds a place for itself in more common and expanded indications by pushing the limits restricting indications. In most publications, positive results of eliminating the mechanical problem in aortic valve are declared almost in all situations of aortic valve surgery. Most cases scaring the surgeons are published with tolerable mortality and morbidities. We believe that AVR with acceptable mortality can be conducted in severe aortic stenosis cases with high pulmonary pressure and accordingly patients might have much more qualified and longer lives.

\section{Limitations of the Study}

The most important limitation of our study was that the difference between pulmonary artery pressure levels and primary pulmonary hypertension could not be determined clearly. This might have been determined by taking biopsy from the patients in pulmonary vascular bed, however, we did not have the chance to execute biopsy. The small samples sum is another limitation.

The difference between the effect of revascularization on myocardial performance and clinical regression in the cases with coronary artery diseases included in the study could not be revealed.

Comparison of our study was conducted in surgical cases. Comparison with the patients receiving medical treatment was realized with the help of the results of medical treatment cases given in the literature.

\section{REFERENCES}

[1] Bonow, R.O., Carabello, B., de Leon Jr., A.C., et al. (1998) ACC/AHA guidelines for the management of patients with valvular heart disease: A report of the American College of Cardiology/American Heart Association Task Force on Practice Guidelines (Committee on Management of Patients With Valvular Heart Disease). Journal of the American College of Cardiology, 32, 
1486-1588. http://dx.doi.org/10.1016/S0735-1097(98)00454-9

[2] Rahimtoola, S.H. (1989) Perspective on valvular heart disease: An update. Journal of the American College of Cardiology, 14, 1-23. http://dx.doi.org/10.1016/0735-1097(89)90047-8

[3] Chizner, M.A., Pearle, D.L. and de Leon Jr., A.C. (1980) The natural history of aortic stenosis in adults. American Heart Journal, 99, 419-424. http://dx.doi.org/10.1016/0002-8703(80)90375-0

[4] Horstkotte, D. and Loogen, F. (1988) The natural history of aortic stenosis. European Heart Journal, 9, 57-64. http://dx.doi.org/10.1093/eurheartj/9.suppl_E.57

[5] Livanainen, A.M., Lindroos, M., Tilvis, R., Heikkila, J. and Kupari, M. (1996) Natural history of aortic valve stenosis of varying severity in the elderly. American Journal of Cardiology, 78, 97-101. http://dx.doi.org/10.1016/S0002-9149(96)00235-4

[6] Bonow, R.O., Carabello, B., de Leon Jr., A.C., et al. (1998) Guidelines for the management of patients with valvular heart disease: Executive summary A report of the American College of Cardiology/American Heart Association Task Force on Practice Guidelines (Committee on Management of Patients with Valvular Heart Disease). Circulation, 98, 1949-1984. http://dx.doi.org/10.1161/01.CIR.98.18.1949

[7] Lund, O., Flo, C., Jensen, F.T., et al. (1997) Left ventricular systolic and diastolic function in aortic stenosis: Prognostic value after valve replacement and underlying mechanisms. European Heart Journal, 18, 1977-1987. http://dx.doi.org/10.1093/oxfordjournals.eurheartj.a01520 $\underline{9}$

[8] Connolly, H.M., Oh, J.K., Orszulak, T.A., et al. (1997) Aortic valve replacement for aortic stenosis with severe left ventricular dysfunction. Prognostic indicators. Circulation, 95, 2395-2400. http://dx.doi.org/10.1161/01.CIR.95.10.2395

[9] Ross Jr., J. and Braunwald, E. (1968) Aortic stenosis. Circulation, 36, IV-61-IV-67.

[10] Schwarz, F., Banmann, P., Manthey, J., et al. (1982) The effect of aortic valve replacement on survival. Circulation, 66, 1105-1110. http://dx.doi.org/10.1161/01.CIR.66.5.1105

[11] Shahbudin, H., Rahimtoola, M.B. and Frye, R.L. (2000) Valvular heart disease. Circulation, 102, IV-24.

[12] Alberico, S., Pompilio, F., Gerard, P.A., Cesare, R., et al. (1998) Ventricular arrhythmias in adult aortic stenosis prevalence, mechanisms, and clinical relevance. Chest, 113, 482-491. http://dx.doi.org/10.1378/chest.113.2.482

[13] Santiga, J.T., Kirsh, M.M., Brady, T.J., et al. (1982) Left ventricular function in patients with ventricular arrhy- thmias and aortic valve disease. Annals of Thoracic Surgery, 35, 152-155.

http://dx.doi.org/10.1016/S0003-4975(10)61452-X

[14] Michel, P.L., Mandagout, O., Vhanian, A., et al. (1992) Ventricular arrhythmias in aortic valve disease before and after valve replacement. Acta Cardiologica, 2, 145-156.

[15] McHenry, M.M., Rice, J., Matlof, H.J. and Flamm Jr., M.D. (1979) Pulmonary hypertension and sudden death in aortic stenosis. British Heart Journal, 41, 463-467. http://dx.doi.org/10.1136/hrt.41.4.463

[16] Connolly, H.M., Oh, J.K., Schaff, H.V., et al. (2000) Severe aortic stenosis with low transvalvular gradient and severe left ventricular dysfunction: Result of aortic valve replacement in 52 patients. Circulation, 101, 1940-1946. http://dx.doi.org/10.1161/01.CIR.101.16.1940

[17] He, G.W., Grunkemeier, G.L., Gately, H.L., Furnary, A.P. and Starr, A. (1995) Up to thirty-year survival after aortic valve replacement in the small aortic root. Annals of Thoracic Surgery, 59, 1056-1062. http://dx.doi.org/10.1016/0003-4975(95)00075-V

[18] Johnson, L.W., Hapanowicz, M.B., Buonanno, C., et al. (1988) Pulmonary hypertension in isolated aortic stenosis Hemodynamic correlations and follow-up. Journal of Thoracic and Cardiovascular Surgery, 95, 603-607.

[19] Tracy, G.P., Proctor, M.S. and Hizny, C.S. (1990) Reversibility of pulmonary artery hypertension in aortic stenosis after aortic valve replacement. Annals of Thoracic Surgery, 50, 89-93. http://dx.doi.org/10.1016/0003-4975(90)90095-N

[20] Snopek, G., Pogorzelska, H., Zielinski, T., et al. (1996) Valve replacement for aortic stenosis with severe congestive heart failure and pulmonary hypertension. Journal of Heart Valve Disease, 5, 268-272.

[21] Malouf, J.F., Enriquez-Sarano, M., Pellikka, P.A., et al. (2002) Severe pulmonary hypertension in patients with severe aortic valve stenosis: Clinical profile and prognostic implications. Journal of the American College of Cardiology, 40, 789-795. http://dx.doi.org/10.1016/S0735-1097(02)02002-8

[22] Pai, R.G., Varadarajan, P., Kapoor, N. and Bansal, R.C. (2007) Aortic valve replacement improves survival in severe aortic stenosis associated with severe pulmonary hypertension. Annals of Thoracic Surgery, 84, 80-85. http://dx.doi.org/10.1016/j.athoracsur.2007.02.094

[23] Varadarajan, P., Kapoor, N., Bansal, R.C. and Pai, R.G. (2006) Clinical profile and natural history of 453 nonsurgically managed patients with severe aortic stenosis. Annals of Thoracic Surgery, 82, 2111-2115. http://dx.doi.org/10.1016/j.athoracsur.2006.07.048

NYHA: New York Heart Association PH: Pulmonary hypertension

AS: Aortic stenosis

AVR: Aortic valve replacement 